\title{
Non-Parametric Mixture Model Based Evolution of Level Sets and Application to Medical Images
}

\author{
Niranjan Joshi • Michael Brady
}

Received: 28 October 2008 / Accepted: 17 August 2009 / Published online: 11 September 2009

(C) Springer Science+Business Media, LLC 2009

\begin{abstract}
We present a novel region-based curve evolution algorithm which has three primary contributions: (i) nonparametric estimation of probability distributions using the recently developed NP windows method; (ii) an inequalityconstrained least squares method to model the image histogram with a mixture of nonparametric probability distributions; and (iii) accommodation of the partial volume effect, which is primarily due to low resolution images, and which often poses a significant challenge in medical image analysis (our primary application area). We first approximate the image intensity histogram as a mixture of non-parametric probability density functions (PDFs), justifying its use with respect to medical image analysis. The individual densities in the mixture are estimated using the recent NP windows PDF estimation method, which builds a continuous representation of discrete signals. A Bayesian framework is then formulated in which likelihood probabilities are given by the non-parametric PDFs and prior probabilities are calculated using an inequality constrained least squares method. The non-parametric PDFs are then learnt and the segmentation solution is spatially regularised using a level sets framework. The log ratio of the posterior probabilities is used to drive the level set evolution. As background to our approach, we recall related developments in level set methods. Results are presented for a set of synthetic and natural images as well
\end{abstract}

The authors thank EPSRC/MRC for the Interdisciplinary Research Consortium MIAS, and Microsoft Research for funding.

N. Joshi $(\bowtie) \cdot$ M. Brady

Department of Engineering Science, University of Oxford, Oxford, UK

e-mail: njoshi@robots.ox.ac.uk

M. Brady

e-mail: jmb@ robots.ox.ac.uk as simulated and real medical images of various anatomical organs. Results on a range of images show the effectiveness of the proposed algorithm.

Keywords Non-parametric probability density functions . Finite mixture models · Curve evolution · Level sets

\section{Introduction}

Image segmentation is a fundamental process that has found application in many fields, ranging from satellite imagery to medical image analysis. The design of a segmentation method typically involves both modelling the data and spatial regularisation of the solution. In this paper, we present a method in which we model the histogram of image intensities using a non-parametric mixture model and regularise the segmentation solution using a level set method. Though the method we present is applicable to any kind of images, we are particularly interested in medical applications, specifically the analysis of MRI (Magnetic Resonance Images) supporting patient management in the case of colorectal cancer.

Finite mixture model (FMM) methods are widely used in image segmentation. They assume that the image is comprised of a finite number of classes and that the intensities of each class follow a certain distribution. Gaussian mixture models (GMMs) are a particularly widely used instance of FMM methods (Zhu and Yuille 1996; Paragios 2000), not least because they have the advantage of simplifying analytical treatment of the problem. While the assumption of class Gaussianity works well in many situations, it does not always, especially in medical image analysis. A more 
generalised approach, which we adopt for the reasons discussed in Sect. 2, is to use non-parametric probability density/mass functions (PDFs/PMFs) to model the individual classes. We refer to our mixture model as a non-parametric mixture model (NPMM) (Joshi and Brady 2005; Joshi 2007) and is described in Sect. 4. Several methods are available to estimate the class non-parametric distributions, including histograms and kernel density approaches (Izenman 1991). By the law of large numbers, a histogram provides a good estimate of a PDF if the number of samples is large. However, this does not hold when PDFs are to be estimated from small regions of an image, for example the local neighbourhoods for which similarity measures such as mutual information or joint entropy need to be calculated. Kernel density methods provide smoother and more accurate estimates with fewer samples than are required for histograms. However, they are sensitive to the setting of the kernel bandwidth parameter, and finding the optimal setting is computationally costly. The NP windows method (Dowson et al. 2008; Joshi 2007), which we use, overcomes both these drawbacks. This method is explained in Sect. 3 .

Level set methods have been used successfully for curve evolution, for example when the curves coincide with the boundaries of the segmentation classes. Because of the implicit nature of level set curve evolution, they can accommodate changes in topology of the curve, and this is of particular relevance in analysing $3 \mathrm{D}$ regions that cross successive slices of a medical image volume. Available level set methods can be broadly divided into three categories: boundarybased methods (Caselles et al. 1997), region-based methods (Zhu and Yuille 1996; Kim et al. 2005), and combination of both (Paragios 2000). We recall some related level set methods published to date in Sect. 5. Then, in light of those previously published methods, we present our algorithm in Sect. 6.

Since our work is motivated primarily by application to medical images, we consider a set of issues that are particularly of importance in that case. One such is the partial volume effect (PVE). Due to the limited resolution of medical images relative to anatomical structures (typically a modern MRI image has resolution of only $1 \mathrm{~mm}^{3}$ ), any single pixel/voxel in the image may contain more than one kind of tissue (Leemput et al. 2003). The resulting intensity value at such a pixel is a linear (MRI) or nonlinear (Positron Emission Tomography) combination of the individual tissue intensities, depending upon the acquisition modality. In certain MRI images as many as $40 \%$ of the total pixels may be affected by the PVE, so it is important to take it into account in the design of any image analysis method. In Sects. 4 and 6, we describe how we take the PVE into account.

\section{Need for Non-Parametric Modelling of Class Distributions}

Most statistical mixture model methods used currently in image analysis (including medical) assume Gaussianity for the individual class PDFs (Zhang et al. 2001; Noe and Gee 2002; Leemput et al. 2003). As noted above, this leads to straightforward analytical treatments. Although, according to physics of MRI, the noise distribution follows a Rician distribution, if the signal to noise ratio (SNR) is sufficiently high it is often closely approximated by a Gaussian distribution (Sijbers 1998). A few methods are also reported to have performed special transformation of intensity values in order to increase the Gaussianity of the data, where the individual class PDFs do not follow Gaussian distribution (Zeydabadi et al. 2004). These observations are generally the rationale for adopting the Gaussian distribution assumption in MR image segmentation. These observations are justifiable for brain MRI, for which RF coil technology is sufficiently advanced to give better quality images and for which the images are intrinsically more or less piecewise smooth. However, the assumptions are far less appropriate for MR images of other parts of the body, not least the liver, breast, and the colorectum, in which we are particularly interested. Due to magnetic field inhomogeneities in the MR scanner, some regions of the image appear 'brighter' or 'darker' than other regions. This is often referred to as the bias field distortion; in the case of colorectal MR images, the bias field distortion is of such a magnitude that a significant residue remains even after applying available preprocessing methods. The segmentation of colorectal MR images involves dividing the image into the colorectum, tumour, and mesorectum. While the tumour tends to be a heterogeneous mass, the mesorectum is chiefly made up of layers of fat, blood vessels, and lymph nodes. Due to this anatomical complexity, a colorectal MR image is not piecewise smooth. See Fig. 1 for an illustration. As a result, the shapes of the distributions of the individual classes are distorted substantially, most often in a way that depends on the particular patient, and is essentially unpredictable. Figure 2 shows a typical example of the class PDFs. In order to take all these effects into account we model the class PDFs using non-parametric distributions. Note, however, that the use of non-parametric distributions makes our segmentation method more generally applicable. For example, consider the simulated Positron Emission Tomographic (PET) image shown in Fig. 3. A differential uptake of radioactive tracer in the heart region gives rise to a bimodal intensity distribution. This would require at least two component distributions in GMMs to describe the intensity spread, but just a single component distribution would suffice with non-parametric distributions. 


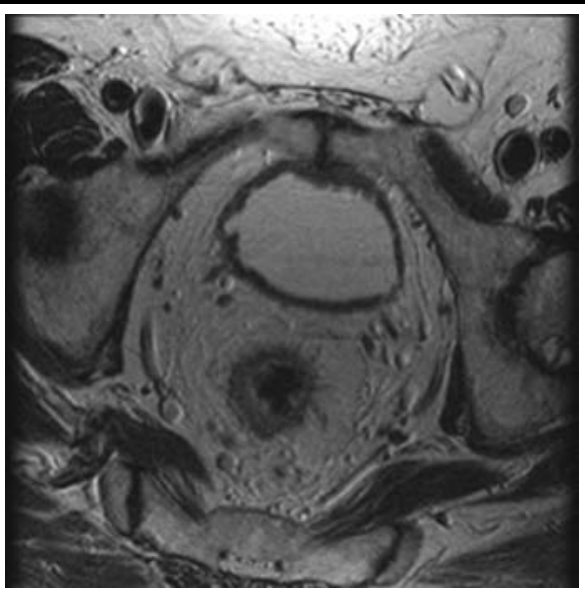

Fig. 1 One slice of a 3-dimensional (3D) colorectal MR image after preprocessing done to remove bias field. Notice the residual bias field in the upper part of the image and a complex heterogeneous nature of the central part of the image

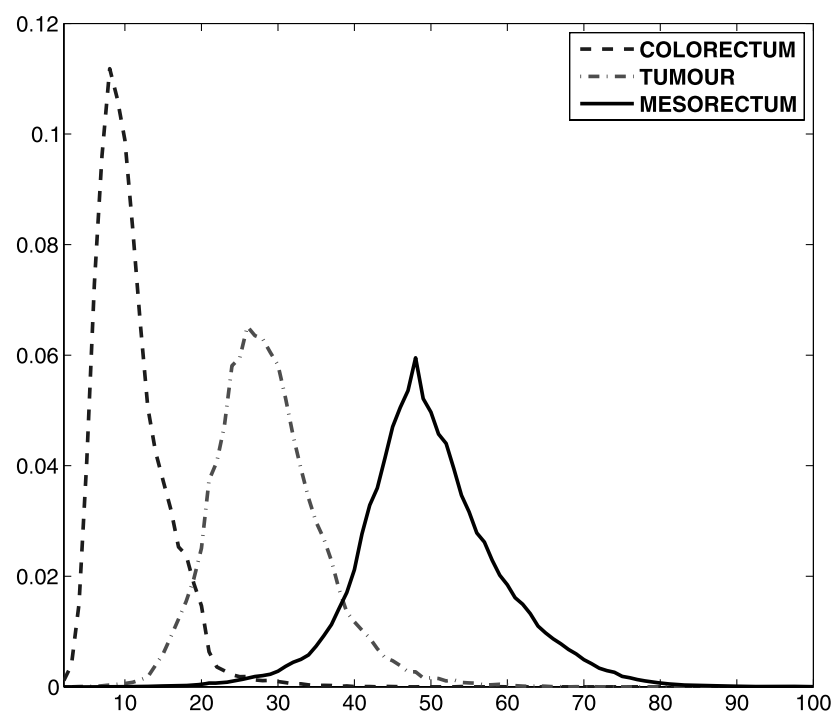

Fig. 2 Histograms of three different tissue classes in a 3D colorectal MR image. Notice the asymmetric nature of the probability distributions. This is mainly because of the residual artifacts such as bias field that remain after preprocessing, and also due to nonconformity to piecewise smooth image assumption

\section{NP-Windows Method for Non-Parametric Estimation of PDFs}

Recently Kadir and Brady proposed (Dowson et al. 2008), and we further developed (Joshi 2007), a method to estimate the PDF of digital signals and images using a continuous representation. The method is based on the observation that a critically or over sampled digital signal can be reconstructed to the original continuous signal if an appropriate interpolation procedure is used. Additional information, determined by the interpolation method, helps improve the resulting PDF estimation. Figure 4 shows a block
Fig. 3 One slice of a simulated

3D Positron Emission

Tomographic (PET) image of lung. Notice differential uptake of radioactive tracer in the heart (black arrow). This gives rise to a bimodal intensity distribution

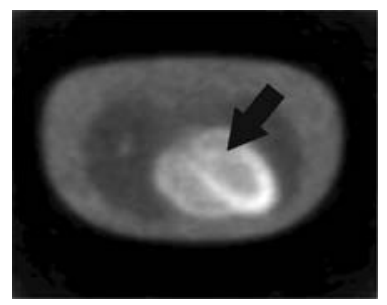

diagram describing the basic idea underlying the NP windows PDF estimator. To illustrate this concept, we describe the simplest case, namely estimating PDFs in 1-dimensional (1D) signals. Consider a 1D signal $y\left(s_{1}\right)$, where the random variable $y$ is a function of the random variable $s_{1}$. Assuming linear interpolation between successive samples, each piecewise linear section of this signal can be represented as $y\left(s_{1}\right)=a s_{1}+b, 0 \leq s_{1} \leq 1$. The transformation formula for the PDF of a monotonic function of a random variable (Papoulis and Pillai 2002) is given by,

$f_{Y}(y)=\frac{1}{\left|\frac{d y}{d s_{1}}\right|} f_{S_{1}}\left(s_{1}\right)$

where $f_{Y}$ and $f_{S_{1}}$ are the PDFs of the random variables $y$ and $s_{1}$ respectively. From (1) and the assumed relationship between $y$ and $s_{1}$, we can conclude that locally

$f_{Y}(y)=\frac{1}{|a|} f_{S_{1}}\left(\frac{y-b}{a}\right), \quad b \leq y \leq a+b$

Further, let us assume that the random variable $s_{1}$ is uniformly distributed between 0 and 1; i.e. $f_{S_{1}}\left(s_{1}\right)=1,0 \leq$ $s_{1} \leq 1$. Then, $f_{S_{1}}\left(\frac{y-b}{a}\right)=1, b \leq y \leq a+b$. It follows that the PDF of a piecewise linear section is given by,

$f_{Y}(y)=\frac{1}{|a|}, \quad b \leq y \leq a+b$

Finally, we obtain an estimate of the PDF $f_{Y}$ of the whole discrete signal, by superposing all the piecewise constant sections of magnitude $\frac{1}{|a|}$. This is illustrated graphically in Fig. 5. We stress that the above was simply to illustrate the method; of course, the method is not restricted just to linear interpolation.

Next, consider a 2-dimensional (2D) image. Again, for illustration, we use bilinear interpolation. Let $Y_{1}$ denote the intensity variable (in this section, all variables will be denoted by upper case letters and particular values will be denoted by lower case letters). The continuous random variables $S_{1}$ and $S_{2}$ denote the positional variables in 2D. We tessellate the image into several sections. The intensity variable $Y_{1}$ is deterministically related to the positional variables over each tessellated section. Typically the relationship is polynomial. Our objective is to find the PDF of the intensity values, given the nature of randomness in the positional variables and the deterministic relationship. Let us 
Fig. 4 Block diagram describing the basic idea behind the NP windows PDF estimator

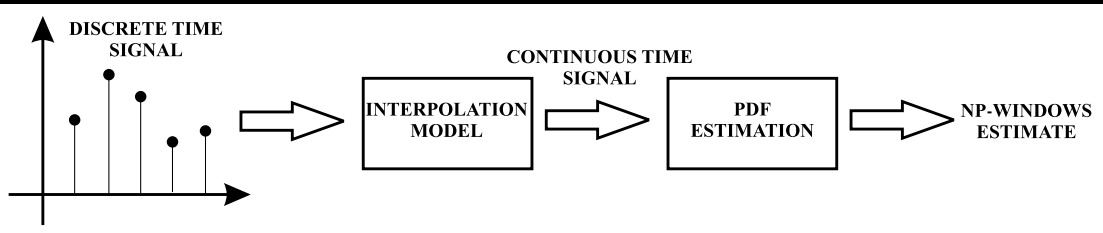

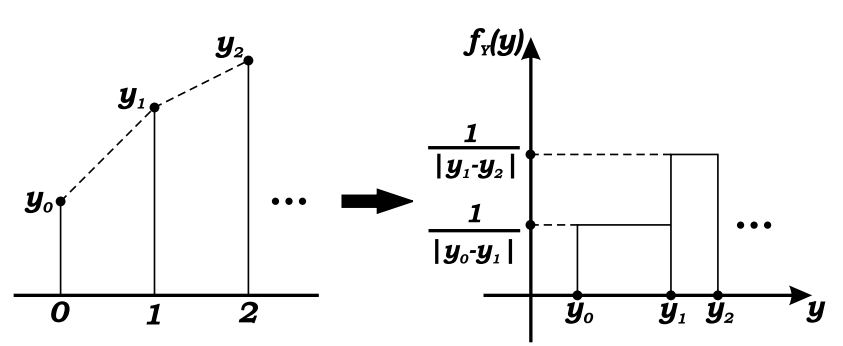

Fig. 5 Samples of a 1-D signal $y$ are shown on the left hand side, intermediate values are interpolated linearly. The corresponding PDF $f_{Y}(y)$ is shown in the form of box functions

suppose that $Y_{1}$ is related to the positional variables by bilinear interpolation over a piecewise section by joining the centres of four neighbouring pixels. This case was proposed in Dowson et al. (2008) and substantially improved in Joshi (2007). Next, it is assumed that the positional variables are uniformly distributed over this piecewise bilinear region i.e. $f_{S_{1}, S_{2}}\left(s_{1}, s_{2}\right)=1$, where $f(\cdot)$ denotes a PDF, and for the spatial region $0 \leq s_{1}, s_{2} \leq 1$ between the centers of four mutually neighbouring pixels. The bilinear relationship between the intensity variable $y_{1}$ and the spatial variable $s_{1}$ and $s_{2}$ gives the following expressions,

$$
\begin{aligned}
& y_{1}\left(s_{1}, s_{2}\right)=a s_{1} s_{2}+b s_{1}+c s_{2}+d, \\
& y_{2}\left(s_{1}, s_{2}\right)=s_{1} \\
& s_{2}\left(y_{1}, y_{2}\right)=\frac{y_{1}-b y_{2}-d}{a y_{2}+c}, \quad s_{1}\left(y_{1}, y_{2}\right)=y_{2}
\end{aligned}
$$

Note that $Y_{2}$ is a dummy variable introduced artificially to make the theoretical development easier, because it renders the Jacobians square. In the above equations, rearranging the first set of equations for $s_{1}$ and $s_{2}$ gives the second set. The joint PDF $f_{Y_{1}, Y_{2}}$ can be calculated by using the transformation formula for functions of random variables (Papoulis and Pillai 2002). In particular,

$$
f_{Y_{1}, Y_{2}}\left(y_{1}, y_{2}\right)=f_{S_{1}, S_{2}}\left(y_{2}, \frac{y_{1}-b y_{2}-d}{a y_{2}+c}\right)|J|
$$

where, $|J|$ is the Jacobian and is equal to $\left|1 /\left(a y_{2}+c\right)\right|$ in this case. Therefore noting the fact that $f_{S_{1}, S_{2}}\left(s_{1}, s_{2}\right)=1$ over the given constraints, we find

$$
f_{Y_{1}, Y_{2}}\left(y_{1}, y_{2}\right)=\frac{1}{a y_{2}+c}
$$

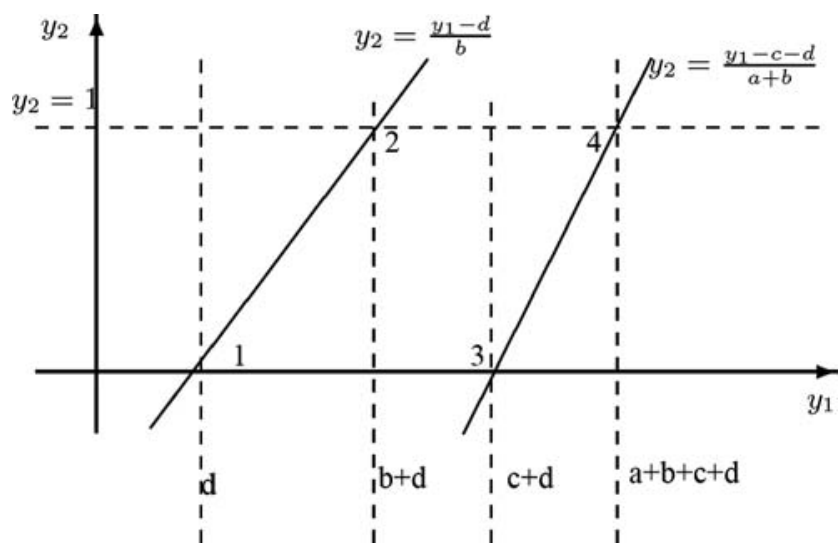

Fig. 6 Shown here is the particular configuration for $\{b, c, d\}>0$, $a<0$ and $a+b>0$, which results into three different integration ranges marked by dashed lines

subject to,

$0 \leq y_{2} \leq 1 \quad$ and $\quad 0 \leq \frac{y_{1}-b y_{2}-d}{a y_{2}+c} \leq 1$

The marginal PDF $f_{Y_{1}}$ is obtained by integrating out the dummy variable $Y_{2}$ over the ranges given in (8). The ranges of integration are shown graphically in Fig. 6. Note that the specific geometry of a configuration is determined by values of the coefficients in (4). In their approach, Dowson et al. (2008) note that 24 such configurations are possible. More specifically, consider the intersection points of the lines in (8). They are marked as points $1,2,3$, and 4 in Fig. 6 . The projections of these points on the $y_{1}$ axis taken in increasing order of their $y_{1}$ value will induce a sequence of four numbers, e.g. in Fig. 6 the sequence is 1234. There are 24 such sequences possible corresponding to the 4 ! ways of arranging the four projected intersection points. We observe (Joshi 2007) that the number of possible combinations depends only on the relative values of the pixel intensities, and not on their absolute values. Therefore even if we shifted the frame of reference of the piecewise bilinear section to a new location/orientation, the PDF of that section would remain the same. In particular, let $v_{1}, v_{2}, v_{3}$, and $v_{4}$ be the values of the corner pixels taken in circular order. Let $v_{\min }$ be the minimum of the four pixels, and associate the origin of the frame of reference with $v_{\min }$. Next, we choose the minimum of the corner pixels in the horizontal or vertical directions, which are neighbours of the "origin" pixel, taken in circular manner. We associate with this pixel the $x_{1}$ axis of the piecewise 


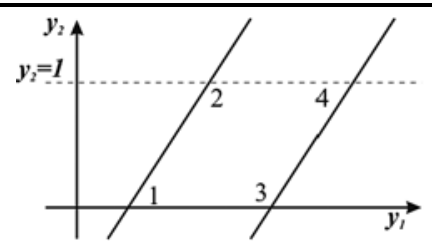

Fig. 7 Three possible configurations after redefining the frame of reference for the bilinear approximation case. As explained in the text, point 4 is the only point that can vary almost arbitrarily. The relative

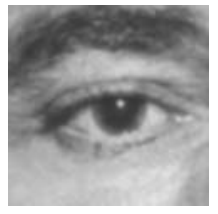

(a) Test image

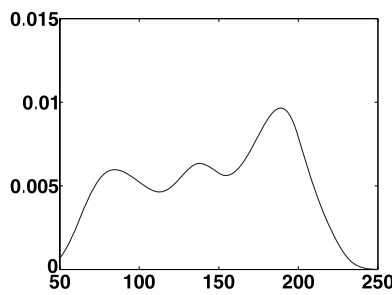

(c) Kernel estimator

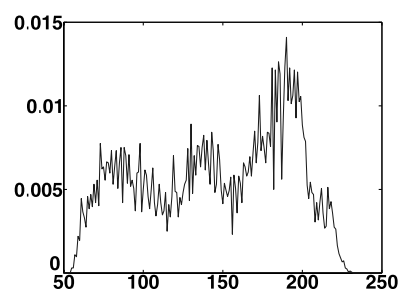

(b) Histogram

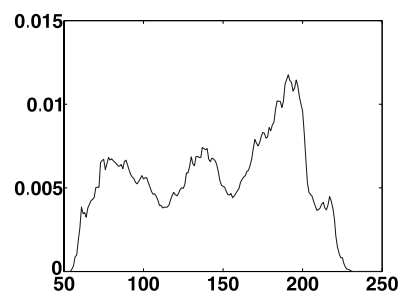

(d) NP windows estimator
Fig. 8 Graphical plots of the various PMF estimates. While the histogram estimate appears very spiked, the kernel estimate tends to be oversmooth for a given kernel bandwidth setting. The NP windows estimate provides a balance between the two

section. With this choice of coordinates, we force the various bilinear interpolation coefficients to satisfy the following constraints: $\{0 \leq b, c, d\},\{b \leq c\}$, and $\{0 \leq a+b+c\}$. This in turn implies that only the coefficient $a$ can vary "almost" arbitrarily. As a consequence of this choice, we are left with only 3 configurations to consider rather than the previous 24. These three configurations are shown graphically in Fig. 7. The PDFs obtained over each tessellated bilinear section are summed and normalised to develop the estimated PDF of the given image.

A few comments are in order before we proceed to apply the NP windows method. First, the NP windows method converts the given discrete signal to its continuous counterpart based on the chosen interpolation method. It follows that the PDF estimate is also continuous. In most applications in image processing we need the PMF of the discrete intensity values and this can be obtained by binning the PDF estimate. The bin width depends on the particular application and in most cases can be set naturally, e.g. for 8-bit gray-scale images it can be set to 1 . Second, the NP window estimate tends to be much smoother than the histogram esti- positions of the remaining points remain fixed. From left to right, the configuration of points sorted in the order of increasing $y_{1}$ coordinate are $1234,1243,1423$

mate particularly for a small number of samples. This is because the NP windows estimate takes into account the information not only at the discrete locations but also the information between locations. Such interpolated information is appropriate as long as the signal is sufficiently smooth, that is band limited. Third, compared to kernel estimators, we notice that in the case of the NP windows method there is no need to set parameters, one needs only to choose an interpolation model. The NP windows method is data driven, which can be seen from the fact that the coefficients in (7) and (8) are all calculated from the input samples. Thus for smooth signals the NP windows method overcomes the drawbacks of both the histogram and the kernel estimators. Figure 8 shows various estimates of PMF for the given test image. The above statements can be visually assessed by looking at the estimates.

\section{NPMM-ICLS Framework}

We have presented a detailed account of this method in Joshi (2007), Joshi and Brady (2005). Let $\mathcal{S}=\{1,2, \ldots, S\}$ be the set of indices for pixels of the image under consideration, where $S$ is the number of pixels in the image. Let $Y=\left\{Y_{1}, Y_{2}, \ldots, Y_{S}\right\}$ be the observed intensity image, and $y_{i}$ be a particular instance of the random variable $Y_{i} . y_{i} \in$ $\mathcal{I}=\left\{0,1, \ldots, I_{\max }\right\}$, where $I_{\max }$ depends upon the number of possible intensity levels in the image; for example in an 8bit grey scale image $I_{\max }=255$. Let $X=\left\{X_{1}, X_{2}, \ldots, X_{S}\right\}$ be the underlying partial volume (PV) segmentation for the image and $x_{i}$ be a particular instance of the random variable $X_{i}$; such that $x_{i} \in \mathcal{L}=\left\{l_{1}, \ldots, l_{K}, \ldots, l_{T}\right\}$ where, $\mathcal{L}$ is the set of all possible tissue class labels, $K$ is the number of pure tissue classes, and $T$ is the total number of tissue classes including partial tissue classes. Note that each $l_{j}$ corresponds to a $K$-dimensional tuple $\mathbf{t}_{\mathbf{j}}=\left[t_{j 1} \ldots t_{j K}\right]^{T}$ of the contributing tissue fractions of each pure tissue, such that $\sum_{k} t_{j k}=1$.

In our case, the value of $K$ is chosen after taking account of anatomical knowledge. To choose the value of $T$, we assume the image model mentioned in Leemput et al. (2003). In their model, the observed intensity image is assumed to result from downsampling a high resolution image, with a downsampling factor $M$. Figure 9 further illustrates this concept. The high resolution image is assumed 


\begin{tabular}{|l|l|}
\hline$\tilde{y}_{1}$ & $\tilde{y}_{2}$ \\
\hline$\tilde{y}_{4}$ & $\tilde{y}_{3}$ \\
$\mathrm{M=2}$
\end{tabular} \begin{tabular}{c}
$y_{1}=$ \\
$\frac{\tilde{y}_{1}+\tilde{y}_{2}+\tilde{y}_{3}+\tilde{y}_{4}}{4}$ \\
\hline
\end{tabular}

Fig. 9 Downsampling image model: (left) high resolution image, (right) low resolution image; here $M=2$

Fig. 10 List of all tissue class labels and corresponding tissue fractions; $K=2$ and $M=2$

\begin{tabular}{|l|l|l|}
\hline & $t_{j 1}$ & $t_{j 2}$ \\
\hline$l_{1}$ & 1.00 & 0.00 \\
$l_{2}$ & 0.00 & 1.00 \\
$l_{3}$ & 0.25 & 0.75 \\
$l_{4}$ & 0.50 & 0.50 \\
$l_{5}$ & 0.75 & 0.25 \\
\hline
\end{tabular}

to be (sufficiently) free of the PVE. With this assumption, the values that $\mathbf{t}_{\mathbf{j}}$ can take are fixed automatically. Figure 10 lists the tissue labels and corresponding tissue fractions for the case $K=2$ and $M=2$. The total number of tissue classes $T$ is decided by the number of pure tissue classes $K$ and the downsampling factor $M$. For instance, in the above example given 2 pure tissue classes and the downsampling factor $M$, we can only have $M^{2}-1$ partial tissue classes as shown in Fig. 10. Hence the total number of tissue classes is $T=\left(M^{2}-1\right)+2=M^{2}+1$. Let $\tilde{Y}=\left\{\tilde{Y}_{1}, \tilde{Y}_{2}, \ldots, \tilde{Y}_{q}, \ldots\right\}$ be the corresponding high resolution image and $\tilde{X}=\left\{\tilde{X}_{1}, \tilde{X}_{2}, \ldots, \tilde{X}_{q}, \ldots\right\}$ be the underlying segmentation. Note that $\tilde{x}_{q}$, a particular instance of the random variable $\tilde{X}_{q}$, is drawn from $\mathcal{L}^{\prime}=\left\{l_{1}, \ldots, l_{K}\right\}$, the set of only pure tissue class labels. As usual, we assume that the random variables $\tilde{Y}_{q}$ are conditionally independent, i.e.

$P(\tilde{Y} \mid \tilde{X})=\prod_{q} P\left(\tilde{Y}_{q} \mid \tilde{X}_{q}\right)$

As shown in Fig. 9, random variable $Y_{1}$ in the observed image is obtained by averaging four corresponding random variables $\tilde{Y}_{1}, \tilde{Y}_{2}, \tilde{Y}_{3}$, and $\tilde{Y}_{4}$. Applying the conditional independence assumption and downsampling model for the image, it can be shown that the PMF

$P\left(Y_{1}=y_{1} \mid X_{1}=x_{1}\right)=4 f\left(4 y_{1}\right)$

where,

$f(\cdot)=P\left(\tilde{Y}_{1} \mid \tilde{X}_{1}\right) * P\left(\tilde{Y}_{2} \mid \tilde{X}_{2}\right) * P\left(\tilde{Y}_{3} \mid \tilde{X}_{3}\right) * P\left(\tilde{Y}_{4} \mid \tilde{X}_{4}\right)$

and $*$ indicates convolution. Therefore, given an estimate of the PMFs for the pure tissue classes in the high resolution image, we can readily calculate the estimates of the basis PMFs of all tissues in the low resolution image.

In the following discussion, for notational convenience, we omit the suffices of the random variables $Y_{i} \mathrm{~s}$ and $X_{i} \mathrm{~s}$. This should not lead to confusion because, in this case, we do not assume spatial correlations, so the position of a pixel in the image does not affect the process of estimating its class label. We note that,

$P(Y)=\sum P(Y \mid X) P(X)$

where $P(Y)$ is the overall intensity distribution, $P(Y \mid X)$ are the basis PMFs corresponding to each pure and partial class, and $P(X)$ is the prior PMF of all the classes. $P(Y)$ and $P(Y \mid X)$ are estimated using the NP-windows method described in the previous subsection. To estimate $P(X)$, we arrange the system of linear equations given in (12) in vector form: $\mathbf{p}_{\mathbf{y}}=\mathbf{P}_{\mathbf{y}, \mathbf{x}} \mathbf{p}_{\mathbf{x}}$ where, $\mathbf{p}_{\mathbf{y}}$ and $\mathbf{p}_{\mathbf{x}}$ are vectors, and $\mathbf{P}_{\mathbf{y}, \mathbf{x}}$ is a matrix whose columns represent the basis PMFs. In order to estimate $\mathbf{p}_{\mathbf{x}}$, we seek a least squares solution to this equation. However, we note that since $\mathbf{p}_{\mathbf{x}}$ represents a PMF; its elements must satisfy the positivity and summability constraints of a PMF. For this reason, we formulate the problem as follows:

$$
\begin{gathered}
\hat{\mathbf{p}}_{\mathbf{x}}=\arg \min _{\mathbf{p}_{\mathbf{x}}} \quad \frac{1}{2}\left(\mathbf{P}_{\mathbf{y}, \mathbf{x}} \mathbf{p}_{\mathbf{x}}-\mathbf{p}_{\mathbf{y}}\right)^{T}\left(\mathbf{P}_{\mathbf{y}, \mathbf{x}} \mathbf{p}_{\mathbf{x}}-\mathbf{p}_{\mathbf{y}}\right) \\
\text { subject to } \quad \mathbf{p}_{\mathbf{x}} \geq \mathbf{0} \quad \text { and } \quad \mathbf{u}^{T} \mathbf{p}_{\mathbf{x}}=1
\end{gathered}
$$

where $\mathbf{u}$ is a vector with all its elements equal to 1 , and $\hat{\mathbf{p}}_{\mathbf{x}}$ is the inequality constrained least square(ICLS) estimate of prior PMF of tissue classes. We adopt a Bayesian framework to obtain the maximum a posteriori (MAP) estimate,

$P(X \mid Y)=\frac{P(Y \mid X) P(X)}{P(Y)}$

where, $P(X \mid Y)$ is posterior PMF of the pure and partial classes. The given pixel belongs to the class for which $P(X \mid Y)$ is maximum. We refer to this new algorithm as a non-parametric mixture model and inequality constrained least squares (NPMM-ICLS) algorithm.

In the above discussion we assumed that the basis PMFs are known. In practice, we often need to learn them using NP windows estimator either by supervised training or by using some other unsupervised method such as K-means clustering. In the work presented here, we adopt the former approach. The next two sections describe how we use curve evolution methods to learn the NPMM.

\section{Curve Evolution Methods}

Curve evolution methods have often been used successfully for image segmentation. Typically, such curves coincide with the boundaries of the segmentation classes. Partial differential equations are frequently used to evolve the curves. The equations are formed following a certain physical principle, such as motion in the normal direction. An alternative approach is to pose the curve evolution problem 
as an energy minimisation problem, and to seek the solution of the corresponding Euler-Lagrange equation. In either case, partial differential equations are solved in an iterative manner to evolve the curve. The curve evolution is based on intrinsic properties of the curve as well as on image based properties. Depending upon the image based properties used, available curve evolution methods may be divided into three categories: region based methods, boundary based methods, and hybrid methods. In this section we review just those methods that are most related to our approach. A more detailed justification would be a paper in itself.

\subsection{Active Contour Snakes}

Active contour snakes were initially proposed by Kass et al. (1988). Since then they have been applied to a wide range of computer vision problems. A snake is an energy minimising curve guided by internal and external constraint forces. The energy of the snake is typically given by,

$$
\begin{aligned}
E[C(r)]= & \int_{0}^{1} E_{\mathrm{int}}[C(r)]+E_{\text {image }}[C(r)] \\
& +E_{\mathrm{ext}}[C(r)] d r
\end{aligned}
$$

where $C(r)$ is the evolving curve parameterised by $r$. The $E_{\text {int }}[\cdot]$ term penalises stretching and bending of the curve, thus keeping it smooth as it evolves under the action of these intrinsic forces. The $E_{\text {image }}[\cdot]$ term attracts the evolving curve towards image features such as bright or dark line, edges, and corners, as well as some interactively provided constraints by the user to guide the curve towards appropriate local minima.

Despite its popularity, the active contour snake approach suffers from two main drawbacks. First, it is highly sensitive to the parameterisation of the curve. Second, the explicit representation of the curve prohibits any topological changes during evolution. Both these drawbacks are overcome by level set methods, which are described next.

\subsection{Level Set Methods}

Level set methods evolve the curve $C$ along the normal direction. However, instead of working directly on this parametric representation of the curve, level set methods embed this curve into a higher dimensional function $\phi$ called a level set function, such that

$C=\{s \in \Omega \mid \phi(s)=0\}$

where, $\Omega$ is image domain. Evolution of the level set function is given by first noting that the curve $C$ is always given by $\phi(C(t), t)=0$, and then by taking the total derivative of the left hand side of this equation we finally get (Sethian 1999)

$\frac{\partial \phi}{\partial t}=-V|\nabla \phi|$

where $V$ is a speed function. Evidently, this equation does not involve any particular parameterisation and, due to the implicit representation of the underlying evolving curve, it can handle changes in topology.

Alternatively, the level set evolution problem can also be posed as energy minimisation problem, just as in the case of active contours. To see this, we first define an energy functional of the form,

$E(\phi)=\int_{\Omega} e\left(s, \phi, \phi_{s}\right) d s$

where $\phi_{s}$ is the derivative of $\phi$ with respect to $s$. Then we observe that to minimise this energy functional amounts to solving the corresponding Euler-Lagrange equation,

$$
\frac{\partial E}{\partial \phi}=\frac{\partial e}{\partial \phi}-\frac{\partial}{\partial s} \frac{\partial e}{\partial \phi_{s}}=0
$$

Then we can evolve the level set function such that (Cremers et al. 2007)

$\frac{\partial \phi}{\partial t}=-\frac{\partial E}{\partial \phi}=-\frac{\partial e}{\partial \phi}+\frac{\partial}{\partial s} \frac{\partial e}{\partial \phi_{s}}$

It now remains to be seen what exactly is the form of the speed function $V$ or the functional $e(\cdot)$ that we used to define the energy minimisation problem. In the next few subsections we will see various popular ways of formulating these functions/functionals.

\subsection{Geodesic Active Contours}

Geodesic active contours (Caselles et al. 1997) are an extension of the classical snakes described above. The energy functional for the method can be given by,

$E[C]=\int_{0}^{1} g(|\nabla I(C(r))|)\left|C^{\prime}(r)\right| d r$

where $I(\cdot)$ is the image, $g(\cdot)$ is a strictly decreasing function such that $g(w) \rightarrow 0$ as $w \rightarrow \infty$. Now noting that $d z=$ $\left|C^{\prime}(r)\right| d r$, where $d z$ is the Euclidean arc length element, the above equation amounts to minimising the weighted length of the curve. The curve evolution equation is obtained via Euler-Lagrange formulation as follows,

$\frac{\partial \phi}{\partial t}=g(|\nabla I|)|\nabla \phi| \kappa-\nabla g(|\nabla I|) . \nabla \phi$

where the curvature $\kappa$ is now computed on the level sets of $\phi$, referred to as the mean curvature. 
Geodesic active contours have two important advantages over the active contours. First, due to the implicit definition of the curve, they can handle the changing topology of the curve as it evolves, and second, they do not depend on any specific parameterisation of the curve. The geodesic active contour method is also an example of boundary based level set methods. However, though popular, it has certain drawbacks. First, it takes into consideration only local information, namely in the form of image gradients. Not surprisingly, the approach works well in cases where images have sharp, well formed edges. However, in the presence of noise, or in the case of less well formed edges, it may fail. Second, it needs to be initialised in a way that is either completely outside or inside the actual boundary.

\subsection{Region Competition}

The region competition algorithm (Zhu and Yuille 1996) minimises a generalised Bayes/Minimum Description Length (MDL) criterion using the variational principle. The following energy functional is formulated,

$$
\begin{aligned}
E\left[C,\left\{\alpha_{i}\right\}\right]= & \sum_{i=1}^{K}\left\{\frac{\mu}{2} \int_{\partial R_{i}} d z\right. \\
& \left.-\iint_{R_{i}} \log P\left(I(\mathbf{s}) \mid \alpha_{i}\right) d \mathbf{s}+\lambda\right\}
\end{aligned}
$$

where the $\alpha_{i}$ 's are parameters of the assumed probability distribution of intensity values for each region $R_{i}, K$ is the total number of regions, $\partial R_{i}$ is the segmentation boundary of the region $R_{i}$, and $\mathbf{s}$ is multi-dimensional space variable. In addition, $\lambda$ is the code length needed to describe the probability distributions and code system for the region $R_{i}$, and it is assumed to be the same for all regions. Minimising this energy amounts to minimising the description length of the segmentation. The curve evolution equation is given by,

$$
\frac{\partial C}{\partial t}=-\mu \kappa \mathcal{N}+\log \left(\frac{P\left(I(C) \mid \alpha_{i}\right)}{P\left(I(C) \mid \alpha_{j}\right)}\right) \mathcal{N}
$$

where, $\mathcal{N}$ is the unit normal vector at a point of the curve.

The region competition algorithm constitutes one way of introducing statistical, region based information into evolving curves. The region information makes the overall curve evolution considerably more robust. However, the algorithm also has a number of intrinsic limitations. First, it ignores any boundary information that may be present. Second, the Gaussian assumption regarding the intensities may prove inadequate in many cases. Third, the region merging step seems unnecessary especially if we use the level set framework which can handle changes in topology naturally.

\subsection{Active Contours Without Edges}

Chan and Vese (2001) proposed a method based on the Mumford-Shah segmentation technique (Mumford and Shah 1990) and the level set method. A special case of the Mumford-Shah functional for segmentation is given by,

$$
\begin{aligned}
E\left(c_{1}, c_{2}, \phi\right)= & \mu \int_{\Omega} \delta_{\epsilon}(\phi(s))|\nabla \phi(s)| d s+v \int_{\Omega} H_{\epsilon}(\phi(s)) d s \\
& +\lambda_{1} \int_{\Omega}\left|I(s)-c_{1}\right|^{2} H_{\epsilon}(\phi(s)) d s \\
& +\lambda_{2} \int_{\Omega}\left|I(s)-c_{2}\right|^{2}\left(1-H_{\epsilon}(\phi(s))\right) d s
\end{aligned}
$$

where $\mu, v, \lambda_{i}$ 's are constants, $\delta_{\epsilon}$ and $H_{\epsilon}$ are regularised versions of the Dirac delta and the Heaviside step functions. In this special case, the solution image $I_{0}$ obtained by minimising the functional is restricted to piecewise constant functions. The associated Euler-Lagrange equation of the above energy functional provides the following update equations,

$$
\begin{aligned}
\frac{\partial \phi}{\partial t}= & {\left[\mu \nabla \cdot\left(\frac{\nabla \phi}{|\nabla \phi|}\right)-v-\lambda_{1}\left(I-c_{1}\right)^{2}\right.} \\
& \left.+\lambda_{2}\left(I-c_{2}\right)^{2}\right] \delta_{\epsilon}(\phi)
\end{aligned}
$$

Note that in the level set update equation, the first term is a curvature based regularisation term, the second term provides a constant ballooning speed term, and the remaining two terms drive the curve towards the actual boundary of the object. The constants $c_{1}$ and $c_{2}$ are simply the sample means of pixel intensities inside and outside the curve.

The Chan and Vese method has many attractive features. For example, due to the region based nature of the edge model used, it is very robust. Very noisy, blurred, and texture images can be segmented satisfactorily. However the statistical information used is limited to first order moments. As a result, the method can be expected to perform unsatisfactorily whenever the intensity distributions have the same first order moment but different higher order moments. A less significant limitation may be complete lack of boundary based edge information in the formulation. Such information is always useful whenever it is available.

\subsection{Geodesic Active Regions}

The geodesic active regions model (Paragios and Deriche 2002; Paragios 2000) unifies boundary and region based information in order to segment an image within the curve evolution framework. In this method, the image histogram is modelled using a Gaussian mixture model (GMM). The number of components/classes is decided using the minimum description length principle for the mixture models. 
Each class is represented by its bounding curve $C$ or equivalently by its interior $R$. The following energy is defined to evolve curves (for simplicity we consider only two classes here),

$$
\begin{aligned}
E(C)= & \alpha \sum_{i=1}^{2} \iint_{R_{i}}-\log \left[P\left(I(\mathbf{s}) \mid R_{i}\right)\right] d \mathbf{s} \\
& +(1-\alpha) \sum_{i=1}^{2} \int_{0}^{1} G\left[P_{B}\left(C_{i}(q) \mid R_{i}\right), \sigma_{B}\right]\left|C_{1}{ }^{\prime}(r)\right| d r
\end{aligned}
$$

where $G(w, \sigma)$ is a Gaussian function with zero mean and variance $\sigma^{2}$. The first term on the right hand side is region based and represents the negative log-likelihood of a pixel belonging to a particular region. The log likelihood is assigned via GMM. The second term is very similar to that in the geodesic active contour method, except for the weighing function. Instead of image gradients used in the original geodesic active contour formulation, here boundary probabilities $P_{B}(\cdot)$ are used. These boundary probabilities are assigned by considering various neighbourhoods of the given pixel. The level set update equation can be given as follows:

$$
\begin{aligned}
\frac{\partial \phi_{1}}{\partial t}= & -\alpha \log \left(\frac{P\left(I \mid R_{1}\right)}{P\left(I \mid R_{2}\right)}\right)\left|\nabla \phi_{1}\right| \\
& +(1-\alpha)\left(G\left[P_{B}(\cdot), \sigma_{B}\right] \kappa_{1}\left|\nabla \phi_{1}\right|\right. \\
& \left.+\nabla G\left[P_{B}(\cdot), \sigma_{B}\right] . \nabla \phi_{1}\right)
\end{aligned}
$$

It can be readily noted that the first term on the right hand side, the negative log-likelihood ratio term is very similar to that in the region competition method. The remaining terms are as in the geodesic active contours method. This is the origin of the name "geodesic" active "regions". This method overcomes many of the drawbacks of the previous methods. However, as we have noted in the previous sections, the Gaussian model used to describe intensities may be inadequate in many cases, not least those medical applications that are our primary interest.

\section{Nonparametric Mixture Model Based Evolution of Curves}

In this section, we develop an algorithm that combines the nonparametric mixture model (NPMM) and the level set curve evolution framework. Some early results of this algorithm were presented in Joshi and Brady $(2007,2008)$. The motivation for our method derives from the geodesic active regions method. The NPMM describes nonparametric statistics of various classes/regions in the image and is therefore a good descriptor of region properties. However, it can not impose spatial regularisation, which, as we have noted, is a desirable component of an image segmentation method. In the level set methods described above, we saw how to impose spatial regularisation by penalising the length of the evolving curve. The curve evolving nature of the level set methods also allows the NPMM algorithm to learn region statistics gradually and from spatially continuous regions. Thus it seems natural to combine the region description model of the NPMM with the level set methods in a framework that is inspired by geodesic active regions. This also enables us to take into account boundary based features, in addition to the region based features. Our level set method will also take into account the partial volume (PV) effect that will be usually useful for segmentation of medical images.

Suppose we have $x_{1}, x_{2}, \ldots, x_{K}$ "pure" classes in our image segmentation problem. Let $C_{1}, C_{2}, \ldots, C_{K}$ be $K$ bounding curves for each pure class and let $R_{1}, R_{2}, \ldots, R_{K}$ be their interior regions. Let $Y$ be the intensity image. Then we build an NPMM as described in Sect. 4. Note that our NPMM, in its most general form, also has partial classes. The NPMM provides us with the following information for each pixel:

- $P\left(x_{k} \mid Y=y\right)=\frac{P\left(Y=y \mid x_{k}\right) P\left(x_{k}\right)}{P(Y=y)}$

the a posteriori probability of the pixel with intensity $Y=y$ belonging to a class $x_{k}$ (note, we have removed the positional description of the pixel for notational convenience).

- $t_{x_{1}}, t_{x_{2}}, \ldots, t_{x_{K}}$ : an estimate of the fractions of pure classes present in the pixel. Given the number of pure tissue classes $K$ and the downsampling factor $M$, the fraction of each pure tissue class in a required partial tissue class can be predetermined as illustrated in Fig. 10.

Now we define an energy functional of our curve evolution method as follows,

$$
\begin{aligned}
E(C)= & \alpha \sum_{i=1}^{K} \iint_{R_{i}}-\log \left(P\left(x_{i} \mid Y\left(s_{1}, s_{2}\right)\right)\right) d s_{1} d s_{2} \\
& +\sum_{i=1}^{K} \int_{0}^{1} g\left(F\left(C_{i}(r)\right)\right)\left|C_{i}^{\prime}(r)\right| d r
\end{aligned}
$$

where $s_{1}$ and $s_{2}$ are positional variables; $g(w)$ is a decreasing function such that as $w \rightarrow \infty, g(w) \rightarrow 0 ; F(\cdot)$ is a boundary feature detector; and $r$ is a parameterisation of the curve. It can be seen that the first term on the right hand side is the a posteriori probability term and provides a region description. The second term is same as in the geodesic active contour and provides boundary description. The boundary weighting term involves the function $F(\cdot)$ which takes a high value if a feature, for example an edge, is present at that point and a low value otherwise. For most researchers in Computer Vision, perhaps the most natural choice for this purpose would be the magnitude of the image gradient. But 
such a choice is often inadequate to describe all types of features. In particular, in the case of colorectal MRI, the boundary of the mesorectum changes in nature from step transitions to delta transitions along its length. This necessitates a feature detector that is not tuned to one specific intensity change model. Fortunately, the above formulation is sufficiently general to accommodate alternative, more general, feature detectors such as local phase (Felsberg and Sommer 2001). Further discussion regarding the use of this feature detector in the context of our method is outside the scope of this paper, but the interested reader is referred to Bond et al. (2007), Joshi (2007). In this paper, we assume that $F=|\nabla Y|$.

In order to evolve the curves $C_{i}$ we embed them in a higher dimensional level set function. We consider $K$ separate level set functions $\phi_{1}, \phi_{2}, \ldots, \phi_{K}$, one for each pure class. For small $K$, it makes sense to use separate level set functions for each class. We evolve each level set function separately. The level set update equations can be found from the corresponding Euler-Lagrange equations, as described above. We obtain the following update equations for each level set function,

$$
\begin{aligned}
\frac{\partial \phi_{k}}{\partial t}= & -\alpha \log \left(\frac{P\left(Y \mid x_{k}\right) P\left(x_{k}\right)}{\sum_{i, i \neq k} P\left(Y \mid x_{i}\right) P\left(x_{i}\right)}\right)\left|\nabla \phi_{k}\right| \\
& +\nabla g(F) . \nabla \phi_{k}+g(F) \kappa_{k}\left|\nabla \phi_{k}\right|
\end{aligned}
$$

The first term on the right hand side involves the log-ratio of a posteriori probabilities. The term gives a positive value if the pixel belongs to the $k$ th class and negative otherwise. Thus the curve expands if it encounters a pixel of the same class and otherwise shrinks. The likelihood probabilities $P\left(Y \mid x_{i}\right)$ are the basis functions of our NPMM, and are estimated using the NP windows method described earlier. The mixture weights or the a priori probabilities are calculated using the NPMM-ICLS algorithm. The remaining terms are as described in the geodesic active contours method in Sect. 5.6.

While the log-ratio term may be sufficient for many image analysis problems, as noted earlier, in medical imaging applications we frequently need to take care of the PVE. We do this as follows. We formulate a second region-based term which inhibits the level sets from entering into the region which NPMM deems to be a partial volume region. This term also serves to couple different level set functions. The modified level set update equation is as follows,

$$
\begin{aligned}
\frac{\partial \phi_{k}}{\partial t}= & -\alpha \log \left(\frac{P\left(Y \mid x_{k}\right) P\left(x_{k}\right)}{\sum_{i, i \neq k} P\left(Y \mid x_{i}\right) P\left(x_{i}\right)}\right)\left|\nabla \phi_{k}\right| \\
& +\beta \sum_{i, i \neq k} t_{x_{i}}\left|\nabla \phi_{k}\right|+\nabla g(F) . \nabla \phi_{k} \\
& +g(F) \kappa_{k}\left|\nabla \phi_{k}\right|
\end{aligned}
$$

where, $\alpha$ and $\beta$ are positive constants, and $t_{x_{k}}$ is fraction of the $k$ th pure tissue class present in that pixel. It can be seen that the newly added term takes positive values outside the region of pure class $k$, thus discouraging the zero level set from entering into that region; on the other hand, it disappears completely in the region of pure class $k$, reducing the equation to the earlier formulation. We refer to this term as the inhibition term, since it inhibits zero level set of one region from entering into other regions.

We now summarise the various steps of our NPMMICLS level set segmentation algorithm.

1. Initialisation: Draw initial curves manually. Calculate initial level set functions using the fast marching method. Calculate initial PMF estimates of pure and partial classes. Set initial mixture weights. Set the feature detector (in the examples shown in this paper we restrict attention to magnitude of image gradients) image.

2. Calculate the $\log$ a posteriori ratio image.

3. Set the inhibition term image (if used).

4. Evolve the level set functions for a small number of iterations.

5. Estimate the PMFs corresponding to pure classes using the NP windows method.

6. Calculate the mixture weights and partial volume fractions using NPMM-ICLS algorithm.

7. Repeat the steps (2) to (6) for a fixed number of iterations.

\section{Results and Discussion}

We have implemented our level set algorithm using the Insight Toolkit (ITK 2005), a C++ library developed specifically to facilitate the efficient development of medical image analysis algorithms. ITK provides a narrowband implementation of the level set evolution method for the following equation (Ibanez et al. 2005),

$\frac{\partial \phi}{\partial t}=-\alpha A(s) . \nabla \phi-\beta D(s)|\nabla \phi|+\gamma Z(s) \kappa|\nabla \phi|$

where $\alpha, \beta$, and $\gamma$ are positive weight parameters, $A(s)$ is called the advection term, $D(s)$ is called the propagation term, and $Z(s)$ is called the spatial modifier term for the mean curvature $\kappa$. This equation is quite general and enables one to implement most of the methods referred to in this paper. It is straightforward to find a one-to-one correspondence between this equation and (30) that we propose to implement. However, we had to modify the basic ITK implementation in order to allow the term $D(s)$ of (31) to vary over iterations, enabling the region properties to be learned gradually. 


\subsection{Results on Natural and Simulated Images}

In this subsection, we present a number of results for a set of natural and simulated images. We assume that each image is to be segmented into foreground and background (i.e. 2 class segmentation). We first assume, that the images are not affected significantly by the PVE. It is very easy to accommodate this assumption in our algorithm. We simply set the downsampling factor $M=1$. This means that for the 2 class segmentation, there are only two pure classes with the class fractions [ $\left[\begin{array}{ll}1 & 0\end{array}\right]$ and $\left[\begin{array}{ll}0 & 1\end{array}\right]$, and there are no partial tissue classes. Refer Fig. 10 for an illustration of how class/tissue fractions can be calculated given the downsampling factor. This setting of $M$ reduces the second term of (30) to $\beta \sum_{i, i \neq k} H\left(\phi_{i}\right)$, where $H(\cdot)$ is the Heaviside step function. Hence the zero level sets of the two pure classes are inhibited from overlapping. The performance of our algorithm in the presence of PVE will be presented in the next subsection. The quality of the results will be judged based on two criteria: visual inspection of the final segmentation of images, and closeness of the NPMM fitted distribution and the overall intensity distribution.

Figure 11 shows a foreground object against a textured background. Both the foreground and background PMFs are unimodal but far from being Gaussian distributions. The NPMM fitted distribution, which is a weighted summation of the individual PMFs, closely matches the overall intensity distribution. Thus our segmentation algorithm not only evolves curves that match the desired boundaries but simultaneously learns the mixture model properly.

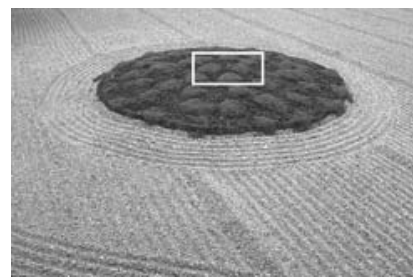

(a)

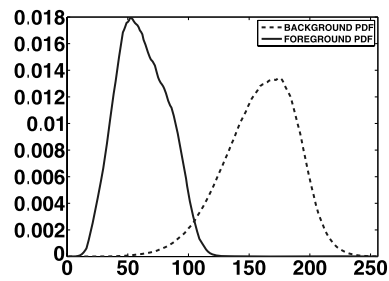

(c)

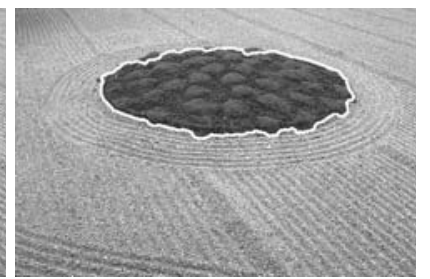

(b)

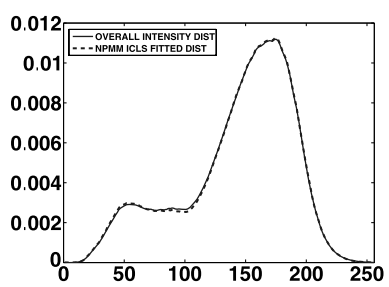

(d)
Fig. 11 Nonparametric mixture model based evolution of level set: results for test image 1 . (a) Initial zero level set contour for the foreground class, (b) final zero level set contour for the foreground class, (c) individual class distributions learnt during the evolution of level set, (d) comparison of NPMM fitted distribution with the overall intensity distribution. Parameter values: $\alpha=1, \beta=0.1, \gamma=1$, number of iterations $=7000$
Unlike test image 1, the individual PMFs in Fig. 12 are not unimodal. Patches of smoothly changing intensities give rise to sharp peaks in the corresponding PMFs. Again the segmentation of the foreground class is satisfactory, except for white tusks. The intensity of the tusks differs markedly from that of rest of the parts. This problem could be overcome by using a higher curvature weight, only at the risk of inclusion of some background part in the foreground; ultimately it is a trade off.

Figure 13 shows a simulated brain MR image. We have amalgamated cerebrospinal fluid and grey matter into a single class, giving a bimodal distribution for that class. This is easily possible due to the nonparametric nature of the distributions involved in the mixture modelling. The segmentation of white matter appears satisfactory. A closer look at the NPMM fitted distribution shows a pronounced mismatch with the overall intensity distribution around the intensity values at the interface of individual PMFs. This is mainly because even if the partial volume effect is present in this image, for the moment we are not taking into consideration (results with PVE will be presented in the next subsection). This also emphasises the importance of explicitly modelling the PVE for medical images.

To compare these results with Gaussian mixture model based level set evolution method, we first obtained GMM model parameters for each test image using the maximumlikelihood expectation maximisation (ML-EM) algorithm described in Noe and Gee (2002). Instead of initialising the GMM algorithm with unsupervised clustering, we use the same interactive procedure as used in the case of the NPMM

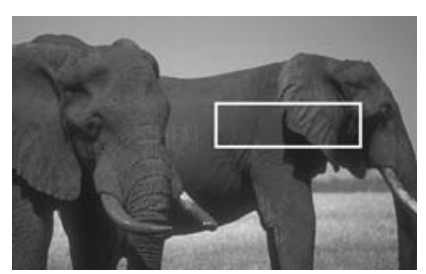

(a)

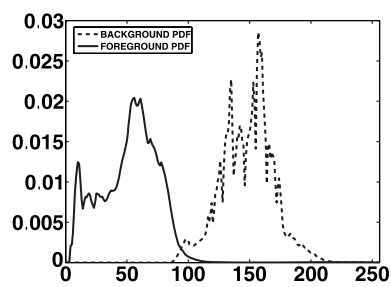

(c)

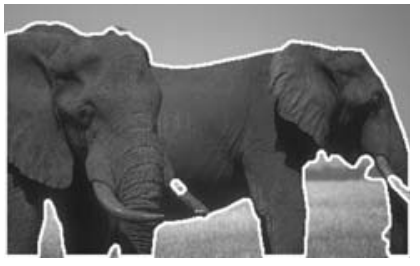

(b)

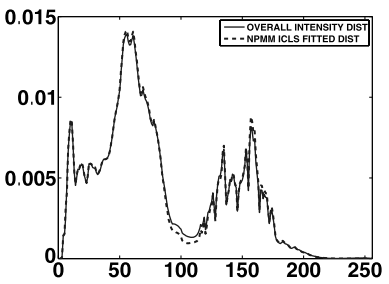

(d)
Fig. 12 Nonparametric mixture model based evolution of level set: results for test image 2. (a) Initial zero level set contour for the foreground class, (b) final zero level set contour for the foreground class, (c) individual class distributions learnt during the evolution of level set, (d) comparison of NPMM fitted distribution with the overall intensity distribution. Parameter values: $\alpha=1, \beta=0.1, \gamma=1$, number of iterations $=7000$ 


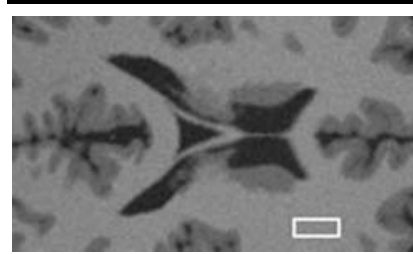

(a)

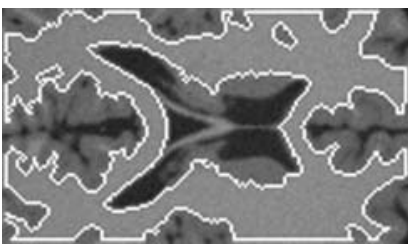

(b)

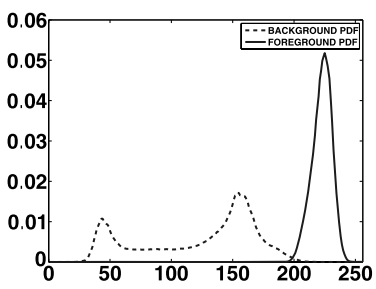

(c)

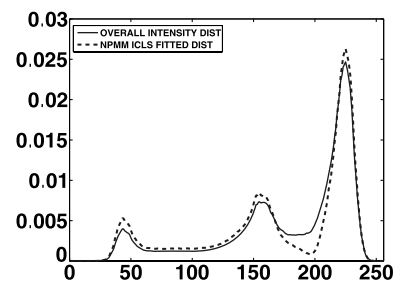

(d)

Fig. 13 Nonparametric mixture model based evolution of level set: results for test image 4. (a) Initial zero level set contour for the foreground class, (b) final zero level set contour for the foreground class, (c) individual class distributions learnt during the evolution of level set, (d) comparison of NPMM fitted distribution with the overall intensity distribution. Parameter values: $\alpha=1, \beta=1, \gamma=1$, number of iterations $=10000$

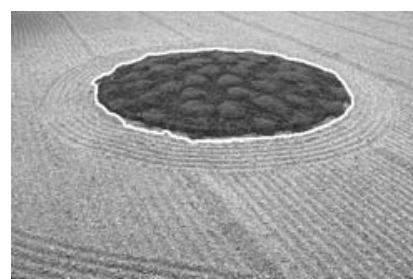

(a)

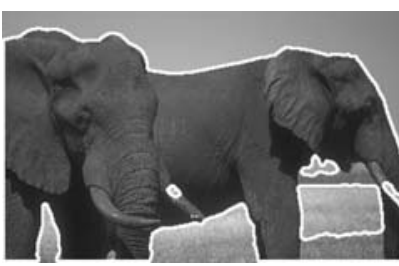

(b)

Fig. 14 Evolution of level set with GMM. The parameter values used were same as that for the corresponding test images shown previously

Table 1 Error norm between the overall intensity distribution and NPMM ICLS fitted distributions using level set framework and that with GMM

\begin{tabular}{lll}
\hline Test image & Error norm: NPMM & Error norm: GMM \\
\hline 1 & $8.5894 \times 10^{-4}$ & $1.66 \times 10^{-2}$ \\
2 & $3.1 \times 10^{-3}$ & $2.27 \times 10^{-2}$ \\
\hline
\end{tabular}

based algorithm to keep the comparison fair. The results are shown in Fig. 14. Comparing them with the corresponding results with NPMM based level set evolution, we can see that the latter method provides significant improvement. Table 1 shows the values of the error norms between the overall intensity distribution and the NPMM ICLS fitted distribution with level set framework and that with GMM. The error norm values are smaller for NPMM based method for each of the test images showing its better model fitting ability.

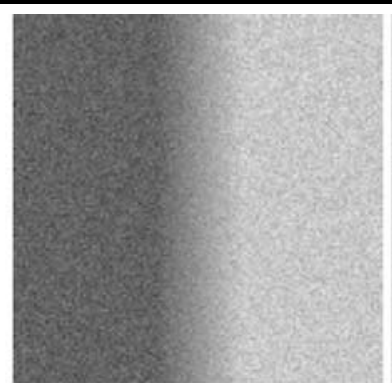

(a)

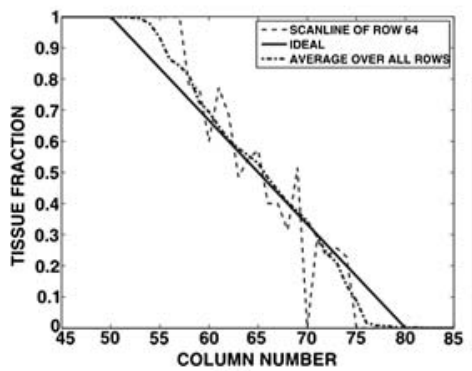

(c)

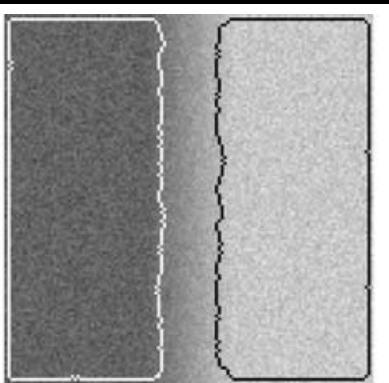

(b)

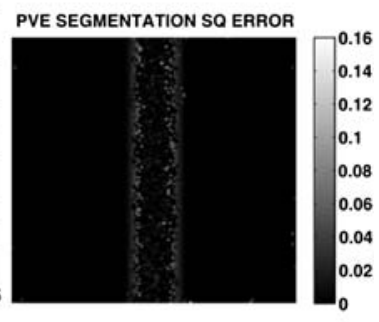

(d)
Fig. 15 Partial volume segmentation of the synthetic image. (a) Synthetic image with peak signal to noise ratio (PSNR) $26 \mathrm{~dB}$. The blurred strip in the middle is the partial volume region as described in the main text. (b) Level set segmentation-note that the middle strip is not occupied by any of the zero level set contours. (c) Scan line of tissue fractions estimated by our algorithm along the row number 64 (dashed line), average scan line taken along all the rows of the tissue fractions estimated by our algorithm (dash-dot line), ideal tissue fractions (solid line). (d) Squared error in estimating partial tissue fractions at each pixel. Parameter values: $\alpha=1, \beta=0.025, \gamma=1$, number of iterations $=7000, M=6$

\subsection{Partial Volume Segmentation}

Figure 15 (a) shows a $128 \times 128$ synthetic image similar to that presented in Noe and Gee (2002). The left and right parts simulate pure tissues. The intensity values of these pure tissues are drawn from Gaussian distributions with means 100 and 200, and with different values of the variances. For example, the image shown in Fig. 15(a) has variance of 100 for both the tissues. The middle part simulates partial/mixed tissues, where we have increased the tissue fractions linearly from 0 to 1 in 30 equal steps. The details of generating the intensity values for these partial tissue pixels with given tissue fractions are described in Noe and Gee (2002). Figure 15(b) shows the partial volume level set segmentation of the synthetic image. Note that neither of the zero level sets enter inside the middle partial volume region because of the overlap inhibition term added in (30). Figure 15(c) compares scan lines of estimated tissue fractions using our algorithm and the ideal tissue fractions. The average scan line matches the ideal scan line closely, especially in the middle part between the columns 55 and 75 . It can be seen that the estimation of tissue fractions is more erroneous towards the boundary of the partial volumed region. This 


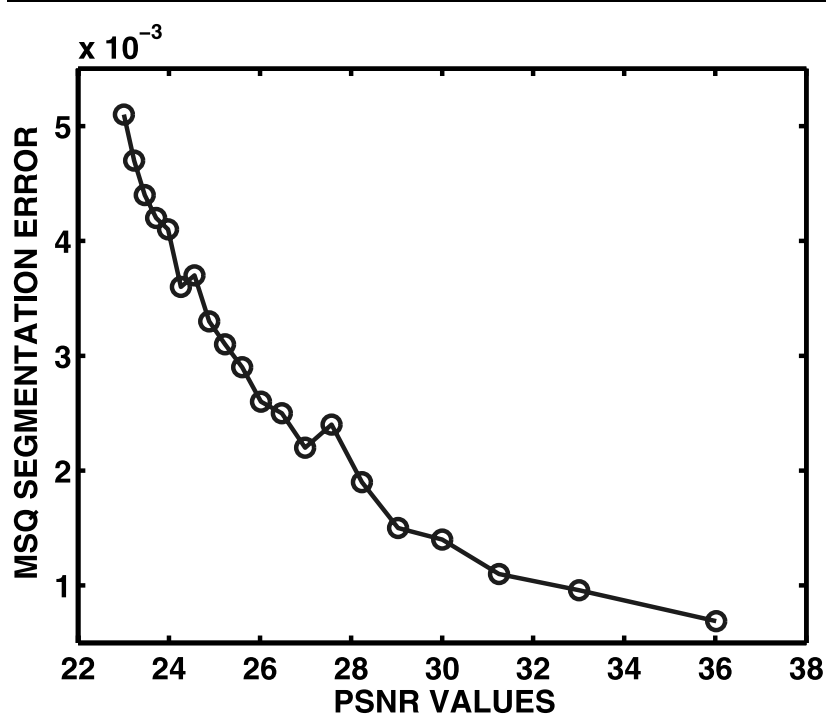

Fig. 16 Performance plot of mean squared (MSQ) error in estimating tissue fractions using our algorithm against PSNR in $\mathrm{dB}$

happens because the overlap inhibition term, which is proportional to the estimated tissue fraction of the other tissue at that location, is weakest near the boundaries. Figure 15(d) shows the squared error values between the estimated and the ideal tissue fractions at each pixel. Figure 16 shows a performance plot of our method against the peak signal to noise ratio (PSNR) in synthetic images. We measured performance using mean squared error in estimating tissue fractions. For this we added pixelwise tissue fraction squared errors, for example as shown in Figure 15(d), and divided this addition by total number of pixels. It can be seen that the performance improves as PSNR values increase. This performance is also better than that reported in Noe and Gee (2002) for the corresponding PSNR values.

\subsection{Simulated Brain MR Image Segmentation}

We obtained simulated T1 weighted brain Magnetic Resonance (MR) images from Brainweb (1997) of varying slice thickness, 3\% noise level, and with no bias field. The aim is to segment brain ventricles, which contain Cerebrospinal Fluid (CSF), from the surrounding white matter. The results of the level set segmentation are shown in Fig. 17. Notice the non-overlapping part between the zero level set contours of the two tissue classes. This part contains partial volume (PV) voxels and a small amount of gray matter voxels which have similar intensity values as the PV voxels. Results are shown for slices of thickness $1 \mathrm{~mm}$ and $3 \mathrm{~mm}$. It can be noticed that the $3 \mathrm{~mm}$ slice has a large number of PV voxels, as expected. In order to quantify the segmentation performance, we calculated the Dice index for each segmentation class. The Dice index compares intersection of the set of segmented pixels with the set of ground truth pixels to the addition of total

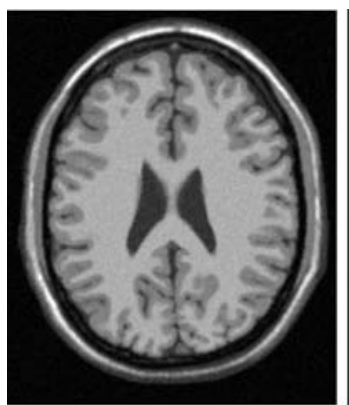

(a)

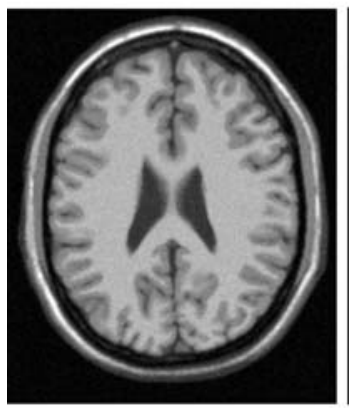

(c)

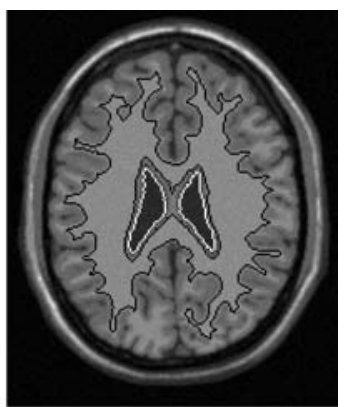

(b)

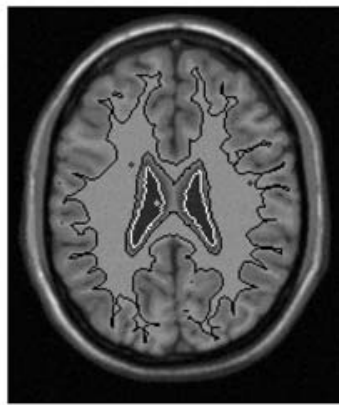

(d)
Fig. 17 Level set segmentation of simulated T1 weighted brain MR images using NPMM-ICLS algorithm. The aim is to segment the ventricles from the surrounding white matter. (a) Slice thinkness of the image is $1 \mathrm{~mm}$. (b) Segmentation of ventricles, notice the non-overlapping part between the zero level set contours of the ventricles and the white matter; this contains partial volumed voxels and a small amount of gray matter. (c) Slice thickness of the image is $3 \mathrm{~mm}$. (d) Corresponding segmentation; notice increased amount of partial volumed region due to larger slice thickness. Parameter values: $\alpha=0$, $\beta=1, \gamma=1$, number of iterations $=1000, M=6$

Table 2 The Dice indices for the segmentation of T1 weighted brain MR images shown in Fig. 17

\begin{tabular}{llll}
\hline Slice thickness & White matter & CSF & PV voxels \\
\hline $1 \mathrm{~mm}$ & 0.9579 & 0.9572 & 0.7396 \\
$3 \mathrm{~mm}$ & 0.9679 & 0.6370 & 0.7038 \\
\hline
\end{tabular}

number of pixels in both the sets. The higher the Dice index, the better the segmentation. The Dice indices for the three segmented parts are shown in Table 2. Segmentation performance for the white matter is much better than the CSF, especially for the $3 \mathrm{~mm}$ slice, due to high signal to noise ratio. Performance to detect the PV voxels is reasonably good, although as noted in Sect. 7.2 this can be improved towards the boundaries of the PV region.

\subsection{Simulated Lung PET Image Segmentation}

A simulated Positron Emission Tomographic (PET) volume was obtained by combining the NCAT phantom (Segars and Tsui 2002) to simulate human anatomy during respiration and a Monte-Carlo based PET simulator PET-SORTEO to 


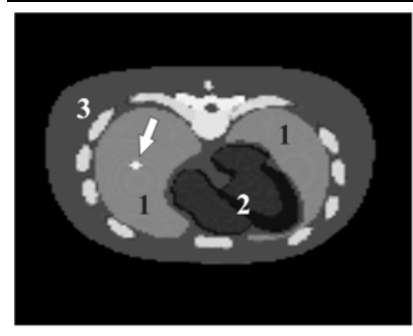

(a)

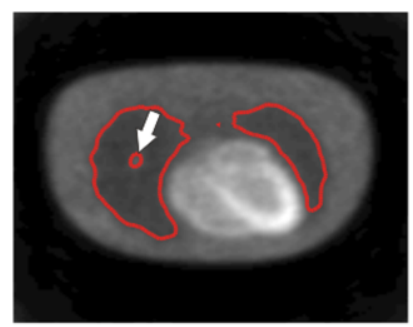

(c)

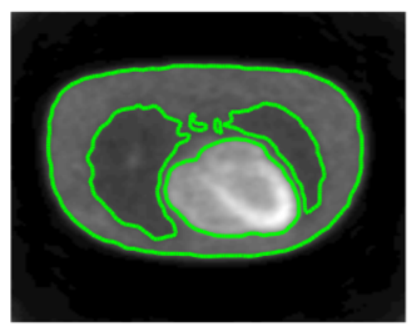

(e)

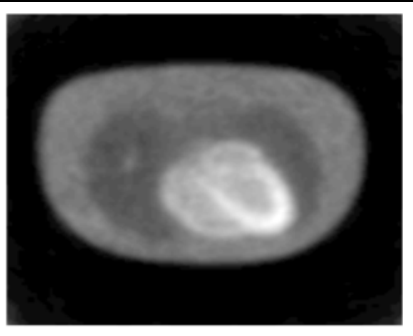

(b)

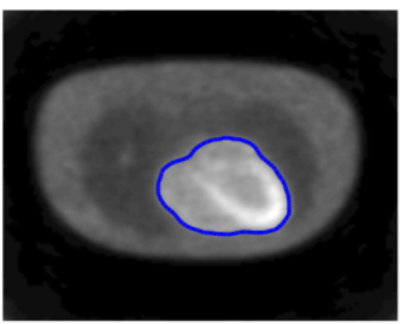

(d)

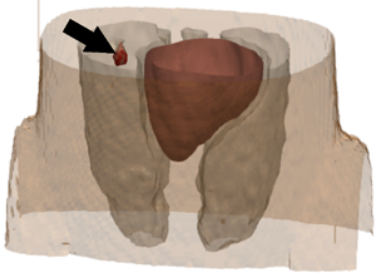

(f)
Fig. 18 (Color online) Level set segmentation of a simulated 3D lung PET volume using NPMM-ICLS algorithm. (a) One slice of the 3D phantom, (thick arrow) shows location of tumour. (b) One slice of the simulated PET volume, notice the blurring of tumour. (c)-(e) Level set segmentation of various tissues, colour code and region numbers: (red, 1) lung; (blue, 2) heart; (green, 3) other tissues; (thick arrow) location of the segmented tumour. (f) 3D zero level set surface reconstruction of various tissues, thick arrow indicates location of the segmented tumour. Parameter values: $\alpha=0, \beta=1, \gamma=1$, number of iterations $=1000, M=3$

simulate an ECAT Exact HR+ 962 scanner (Reilhac et al. 2004). A small spherical lesion was added to the lung region using the NCAT phantom software. The radiotracer activity of the lesion was set to 8 times that of the lungs. The 3D Ordered Subset Expectation Maximisation (OS-EM) method was used to reconstruct the simulated PET volume along with attenuation correction and $4 \mathrm{~mm}$ Gaussian filtering. The size of the reconstructed PET volume was $128 \times 128 \times$ 61 with the voxel dimensions $2 \mathrm{~mm} \times 2 \mathrm{~mm} \times 2.425 \mathrm{~mm}$. Please refer to Bai and Brady (2009) for further detail about the procedure used to generate the simulated data.

Figures 18 (a), (b) show one slice of the 3D NCAT phantom and the corresponding slice of the simulated PET volume. We notice blurred boundaries between the tissues due to low resolution of the image. For this reason we do not use the boundary based term in the level set equation for this experiment. We aim to segment the PET volume into three tissue classes, namely lung, heart, and all the other tis-
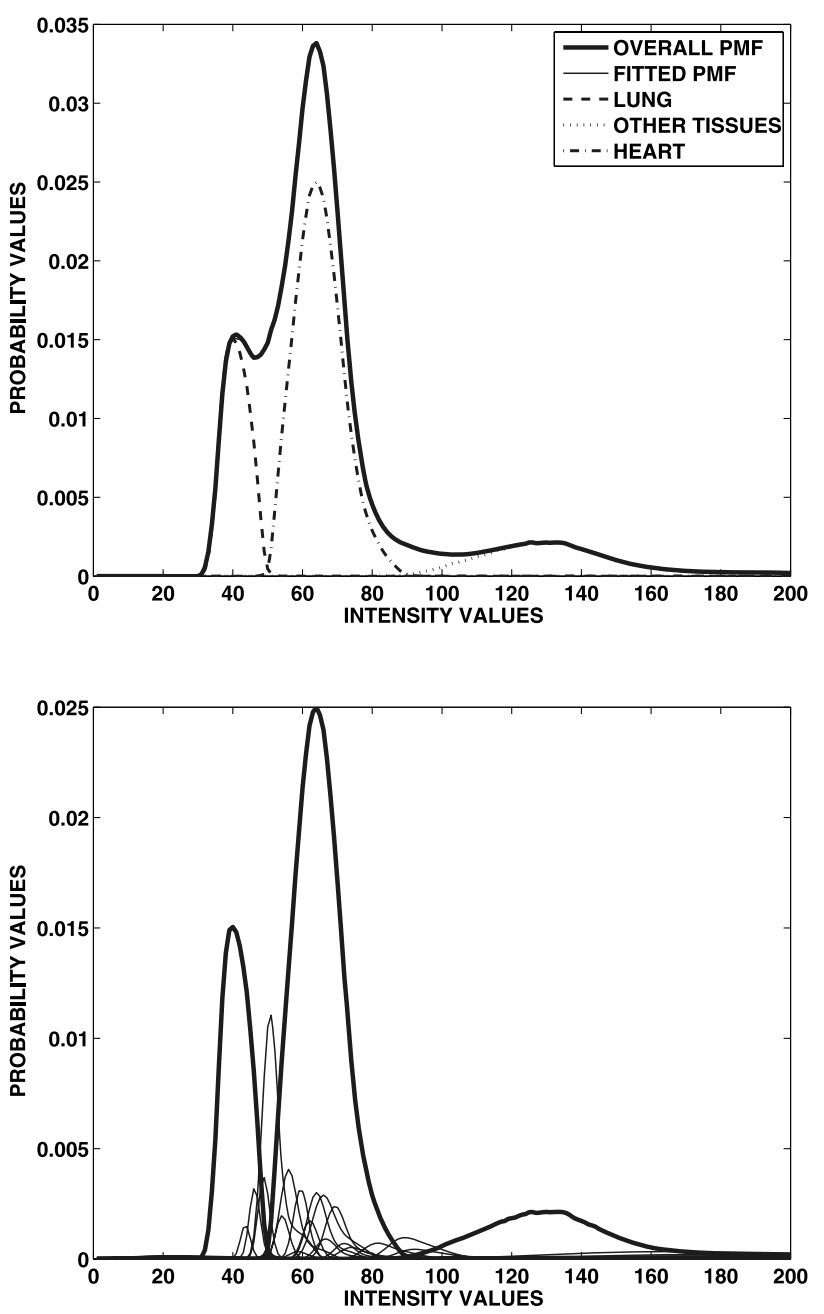

Fig. 19 (Top) Various distributions for the 3D lung PET volume, (bottom) pure tissue distributions in bold solid lines and partial tissue distributions in thin solid lines. Note that each individual distribution has been weighted appropriately

sues as a single segmentation class. The tumour lesion separates out as an isolated non-lung region in the lung tissue class. Figures 18 (c)-(e) show segmentation of three tissue classes. Visual inspection of the segmentation results shows some misclassified regions, such as near the spinal chord in Fig. 18 (e); but otherwise the segmentation is satisfactory, in fact significantly beyond what is current in medical image analysis. The Dice indices for various tissue classes are: Lung $=0.9065 ;$ Heart $=0.859$; Other tissues $=0.7368$; Tumour lesion $=0.5546$. We notice that the Dice index for the main three tissue classes is reasonably good considering the low resolution of the data. The Dice index for tumour lesion is low, however if we compare size of the tumour lesion in voxels (ground truth $=62$ voxels, segmented lesion $=57$ voxels) it is quite comparable. A possible reason for this to happen may be the blurring processes occurring during the reconstruction of the data. Finally, Fig. 18 (f) shows 3D reconstruction of the segmented tissues. 


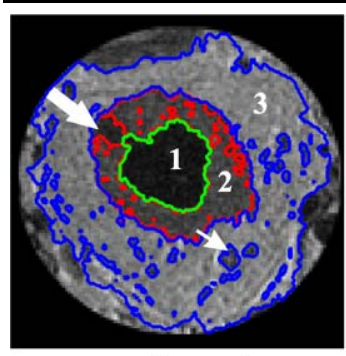

(slice 1)

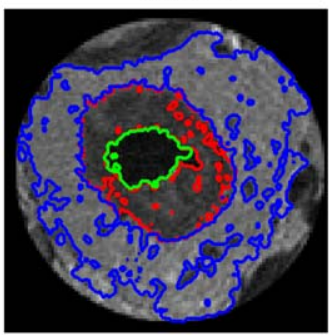

(slice 3)

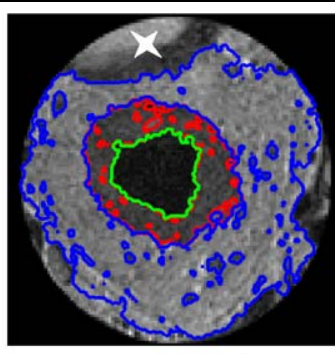

(slice 2)

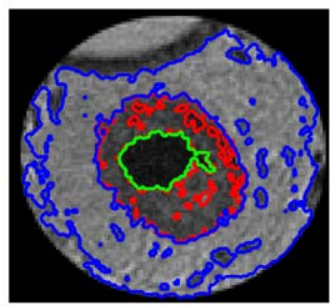

(slice 4)
Fig. 20 (Color online) Level set segmentation of four colorectal MR image slices using NPMM-ICLS algorithm. Colour code and region number: (green, 1) colorectum; (red, 2) tumour; (blue, 3) mesorectum. (thick arrow): partial volumed region, see the text for further details; (thin arrow): blood vessel/lymph node; (star): outside region. Parameter values: $\alpha=1, \beta=1, \gamma=1$, number of iterations $=70, M=4$

Figure 19 shows various distributions involved in the segmentation of the 3D PET volume. The NPMM-ICLS fit to the overall PMF is quite good. Also notice skewed nature of the distributions corresponding to the other tissues and the heart.

\subsection{MR Image Segmentation}

We also tested our algorithm on a set of real medical images. The images used were colorectal magnetic resonance (MR) images. The image acquisition protocol comprised axial small field of view T2 weighted MR images $(\mathrm{TE}=90 \mathrm{~ms}$, $\mathrm{TR}=3500-5000 \mathrm{~ms}, \alpha=90 \mathrm{deg}$, slice thickness $=3 \mathrm{~mm}$ ) acquired using a clinical 1.5T MRI machine. We assumed three pure tissue classes namely: colorectum, tumour, and mesorectum. The original images contained whole abdominal section, which were then manually cropped to contain only region of interest.

Figure 20 shows the results of our new level set algorithm on four consecutive slices of a 3D colorectum MR image. We notice that all three solutions (corresponding to each level set function) are spatially continuous. This is due to the curvature based regularisation. At the same time there are certain regions which do not lie inside any of the zero level sets. This happens because the NPMM deems those regions to be partial volume regions, and therefore the inhibition force stops the zero level sets entering into these regions. These regions can be further studied using more accurate pixel-by-pixel image analysis methods such as the
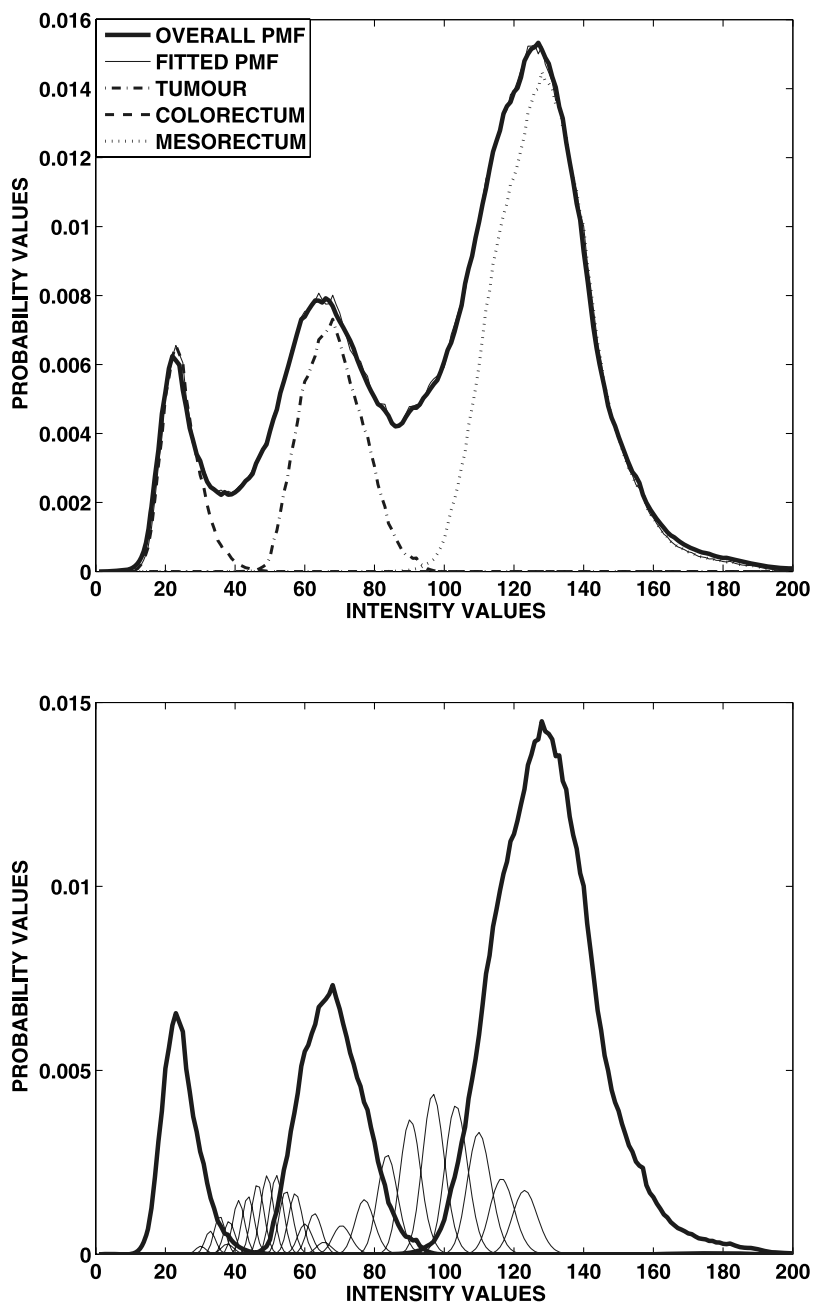

Fig. 21 (Top) Various distributions for the first slice of the colorectal MR images, (bottom) pure tissue distributions in bold solid lines and partial tissue distributions in thin solid lines. Note that each individual distribution has been weighted appropriately

NPMM-ICLS algorithm. For example, the region shown by the "thick arrow" in Fig. 20 (slice 1) is a partial volumed region between the colorectum and the tumour classes. In this case partial voluming happens between two consecutive slices which are $3 \mathrm{~mm}$ thick. We also observe that there are small "holes" in the outermost segmentation class (mesorectum) as indicated by the "thin arrow" in Fig. 20 (slice 1). Some of them are present in the consecutive slices whereas others disappear. Based on their pattern of appearance, one can either classify them as tubular (blood vessels) or ellipsoidal (lymph nodes) structures. This classification can potentially help deciding the stage to which colorectal caner has grown. Few, if any, methods have been proposed so far to detect sentinel lymph nodes reliably; we are currently investigating whether our new method could be helpful for that task (Bond et al. 2007). Figure 21 shows various distributions involved in driving the level set solution. The NPMM- 
ICLS fitted PMF matches very closely with the overall intensity PMF, which shows better modelling of the intensity values. The individual class PMFs are also shown and have non-Gaussian shapes. Note that the region outside the boundary of the mesorectum, e.g. as indicated by the "star" in Fig. 20 (slice 2), is of no clinical importance in the analysis of colorectal cancer images. Hence anything outside the outermost boundary of the mesorectum is not considered in our image analysis.

\section{Conclusions}

In this paper we have presented a novel level set evolution method. We consider that there are three primary contributions: (i) non-parametric estimation of probability distributions using the recently developed NP-windows method, (ii) NPMM-ICLS modelling of the image histogram, and (iii) accommodation of the partial volume effect. While the utility of each of the individual method has been shown already in various image analysis applications, we show here that they can be gainfully combined for curve evolution purposes. The NPMM provides a way of describing intensity values of various classes and the level set framework provides spatial continuity to the segmentation solution. We also saw that boundary features such as the magnitude of image gradient can be easily incorporated into the new level set framework. Results were presented for increasingly difficult test images. They show the effectiveness of our curve evolution algorithm in terms of segmenting images and also in terms of learning the corresponding mixture model of intensity values. The results also emphasise the need to model explicitly the partial volume effect (PVE), particularly in the case of medical images. We are currently exploring a number of avenues to develop further this concept of NPMM level set evolution. First, we have begun experimenting with different boundary feature detectors such as local phase, which can be experimented instead of magnitude of gradient to improve segmentation especially for colorectal cancer MR images showed in the previous section (Bond et al. 2007; Joshi 2007). Second, throughout the previous section, we initialised level set segmentation manually. However, an unsupervised method of learning non-parametric distributions, while at the same time segmenting images without manual initialisation, can be developed on lines similar to those described by Rathi et al. (2006), Michailovich et al. (2007). Currently we are working on such an unsupervised approach.

\footnotetext{
Acknowledgements The authors thank Wenjia Bai for providing 3D simulated PET data. They thank the anonymous reviewers for their comments, which have substantially improved the paper. They also thank Prof. Tony Hey and Prof. Andrew Blake of Microsoft Research for funding and for providing useful comments.
}

\section{References}

Bai, W., \& Brady, M. (2009). Regularized b-spline deformable registration for respiratory motion correction in pet images. Physics in Medicine and Biology, 54, 2719-2736.

Bond, S., Joshi, N. B., Petroudi, S., \& Brady, M. (2007). Estimating the mesorectal fascia in MRI. In: The proceedings of information processing in medical imaging-IPMI.

Brainweb (1997). http://www.bic.mni.mcgill.ca/brainweb/.

Caselles, V., Kimmel, R., \& Sapiro, G. (1997). Geodesic active contours. International Journal of Computer Vision, 22(1), 61-79.

Chan, T. F., \& Vese, L. A. (2001). Active contours without edges. IEEE Transactions on Image Processing, 10(2), 266-277.

Cremers, D., Rousson, M., \& Deriche, R. (2007). A review of statistical approaches to level set segmentation: integrating colour, texture, motion, and shape. International Journal of Computer Vision, 72(2), 195-215.

Dowson, N., Kadir, T., \& Bowden, R. (2008). Estimating the joint statistics of images using non-parametric windows with application to registration using mutual information. IEEE Transactions on Pattern Analysis and Machine Intelligence, 30(10), 1841-1857.

Felsberg, M., \& Sommer, G. (2001). The monogenic signal. IEEE Transactions on Signal Processing, 12, 3136-3144.

Ibanez, L., Schroeder, W., Ng, L., \& Cates, J. (2005). The insight segmentation and registration toolkit software guide, version 2.4. Available at www.itk.org.

ITK (2005). The insight segmentation and registration toolkit. Available at www.itk.org.

Izenman, A. J. (1991). Recent developments in non-parametric density estimation. Journal of the American Statistical Association, 86(413), 205-224.

Joshi, N. B. (2007). Non-parametric probability density function estimation for medical images. DPhil thesis, University of Oxford.

Joshi, N. B., \& Brady, M. (2005). A non-parametric mixture model for partial volume segmentation of MR images. In: The proceedings of British machine vision conference-BMVC.

Joshi, N. B., \& Brady, M. (2007). Non-parametric mixture model based evolution of level sets. In: The proceedings of international conference on computing: theory and applications-ICCTA (pp. 618622).

Joshi, N. B., \& Brady, M. (2008). Non-parametric mixture model based evolution of level sets. In: Chanda B., Murthy C.A. (Eds.), Advances in intelligent information processing: tools and applications, World Scientific Publishing, Indian Statistical Institute, India, Platinum Jubilee Series.

Kass, M., Witkin, A., \& Terzopoulos, D. (1988). Snakes: active contour models. International Journal of Computer Vision, 321-331.

Kim, J., Fisher, J., Yezzi, A., Cetin, M., \& Willsky, A. (2005). A nonparametric statistical method for image segmentation using information theory and curve evolution. IEEE Transactions on Image Processing, 14(10), 1486-1502.

Leemput, K. V., Maes, F., Vandermuelen, D., \& Suetens, P. (2003). A unifying framework for partial volume segmentation of brain mr images. IEEE Transactions on Medical Imaging, 22, 105-119.

Michailovich, O., Rathi, Y., \& Tannenbaum, A. (2007). Image segmentation using active contours driven by the Bhattacharyya gradient flow. IEEE Transactions on Image Processing, 16(11), 27872801.

Mumford, D., \& Shah, J. (1990). Boundary detection by minimizing functionals, I*. In: Ullman S., Richards W. (Eds.), Image understanding (Chap. 2).

Noe, A., \& Gee, J. C. (2002). Efficient partial volume tissue classification in MRI scans. In LNCS: Vol. 2488. Proceedings of the 5th international conference on medical image computing and computer-assisted intervention-MICCAI 2002 (pp. 698-705). Berlin: Springer. 
Papoulis, A., \& Pillai, S. U. (2002). Probability, random variables and stochastic processes. New York: McGraw-Hill.

Paragios, N. (2000). Geodesic active regions and level set methods: contributions and applications in artificial vision. $\mathrm{PhD}$ thesis, University of Nice Sophia Antipolis/INRIA, France.

Paragios, N., \& Deriche, R. (2002). Geodesic active regions: a new framework to deal with frame partition problems in computer vision. Journal of Visual Communication and Image Representation, 13, 249-268.

Rathi, Y., Michailovich, O., Malcolm, J., \& Tannenbaum, A. (2006). Seeing the unseen: segmenting with distributions. In: International conference on signal and image processing (SIP).

Reilhac, A., Lartizien, C., Costes, N., Sans, S., Comtat, C., Gunn, R., \& Evans, A. (2004). Pet-sorteo: a Monte Carlo based simulator with high count rate capabilities. IEEE Transactions on Nuclear Science, 51, 46-52.

Segars, W., \& Tsui, B. (2002). Study of the efficacy of respiratory gating in myocardial spect using the new 4d NCAT phantom. IEEE Transactions on Nuclear Science, 49, 675-679.
Sethian, J. A. (1999). Level set methods and fast marching methods. Cambridge: Cambridge University Press.

Sijbers, J. (1998). Signal and noise estimation from magnetic resonance images. PhD thesis, University of Antwerp, Belgium.

Zeydabadi, M., Zoroofi, R. A., \& Soltanian-Zadeh, H. (2004). Multiresolution automatic segmentation of T1-weighted brain MR images. In: Proceedings of IEEE international symposium on biomedical imaging conference (pp. 165-168).

Zhang, Y., Brady, M., \& Smith, S. (2001). Segmentation of brain MR images through a hidden Markov random field model and the expectation maximization algorithm. IEEE Transactions Medical Imaging, 20, 45-57.

Zhu, S. C., \& Yuille, A. (1996). Region competition: unifying snakes, region growing, and Bayes/MDL for multiband image segmentation. IEEE Transactions on Pattern Analysis and Machine Intelligence, 18(9), 884-900. 